\title{
Effective scheme to determine accurate defect formation energies and charge transition levels of point defects in semiconductors
}

\author{
Cang Lang Yao, ${ }^{1}$ Jian Chen Li, ${ }^{1}$ Wang Gao, ${ }^{1, *}$ Alexandre Tkatchenko, ${ }^{2}$ and Qing Jiang ${ }^{1}$ \\ ${ }^{1}$ Key Laboratory of Automobile Materials (Jilin University), Ministry of Education, Department of Materials Science and Engineering, \\ Jilin University, Changchun 130022, China \\ ${ }^{2}$ Physics and Materials Science Research Unit, University of Luxembourg, L-1511 Luxembourg \\ (Received 10 January 2017; revised manuscript received 26 September 2017; published 26 December 2017)
}

\begin{abstract}
We propose an effective method to accurately determine the defect formation energy $E_{\mathrm{f}}$ and charge transition level $\varepsilon$ of the point defects using exclusively cohesive energy $E_{\text {coh }}$ and the fundamental band gap $E_{\mathrm{g}}$ of pristine host materials. We find that $E_{\mathrm{f}}$ of the point defects can be effectively separated into geometric and electronic contributions with a functional form: $E_{\mathrm{f}}=\chi E_{\mathrm{coh}}+\lambda E_{\mathrm{g}}$, where $\chi$ and $\lambda$ are dictated by the geometric and electronic factors of the point defects ( $\chi$ and $\lambda$ are defect dependent). Such a linear combination of $E_{\mathrm{coh}}$ and $E_{\mathrm{g}}$ reproduces $E_{\mathrm{f}}$ with an accuracy better than $5 \%$ for electronic structure methods ranging from hybrid densityfunctional theory (DFT) to many-body random-phase approximation (RPA) and experiments. Accordingly, $\varepsilon$ is also determined by $E_{\mathrm{coh}} / E_{\mathrm{g}}$ and the defect geometric/electronic factors. The identified correlation is rather general for monovacancies and interstitials, which holds in a wide variety of semiconductors covering $\mathrm{Si}, \mathrm{Ge}$, phosphorenes, $\mathrm{ZnO}, \mathrm{GaAs}$, and $\mathrm{InP}$, and enables one to obtain reliable values of $E_{\mathrm{f}}$ and $\varepsilon$ of the point defects for RPA and experiments based on semilocal DFT calculations.
\end{abstract}

DOI: 10.1103/PhysRevB.96.245203

\section{INTRODUCTION}

Point defects are intrinsic in all real materials, and they often determine the electronic and optical properties of semiconductors [1]. Defect stabilities have been extensively studied, with a vast accumulated understanding that has profoundly shaped the semiconductor industry. However, identifying concentrations and types of defects still remains a fundamental challenge due to the absence of a practical and accurate scheme for determining accurately defect formation energies and charge transition levels.

The commonly used (semi)local-density functionals vastly underestimate defect formation energies due to the electron self-interaction error (SIE) [2,3]. Hybrid functionals mitigate the electron SIE; however, they generate results evidently different from high-level many-body calculations [4-14]. In principle, accurate defect formation energies could be obtained with many-body methods: $G W$, quantum Monte Carlo $(\mathrm{QMC})$, and random-phase approximation (RPA). However, high-throughput calculations based on these methods are not realistic, especially in point-defect systems, for which large supercells are required. In addition, these many-body methods still adopt severe assumptions (e.g., pseudopotentials and fixed node approximation in QMC [9-11]) that could introduce sizable uncertainties in the obtained results.

It is crucial to develop efficient theories to understand point defects from a more phenomenological perspective. Since defect formation energies $E_{\mathrm{f}}$ and charge transition levels $\varepsilon$ of a certain type are a function of properties of the host bulk materials, models that obtain $E_{\mathrm{f}}$ and $\varepsilon$ from the easily computable bulk properties of host materials become a natural choice. Ramprasad et al. adopted the valence bandwidth of host nonmetal material as the index for $E_{\mathrm{f}}$, observing a linear correlation between them

\footnotetext{
*wgao@jlu.edu.cn
}

[15]. However, the slope and intercept in their scheme are elusive, limiting the understanding of the underlying mechanism.

Here we propose an efficient model to determine $E_{\mathrm{f}}$ and $\varepsilon$ of the point defects in semiconductors from the bulk properties of the host materials, namely, the cohesive energy $E_{\text {coh }}$ and the fundamental band gap $E_{\mathrm{g}}$. We find that $E_{\mathrm{f}}$ of each neutral and charged point defect can be effectively separated into geometric and electronic contributions with the following formula: $E_{\mathrm{f}}=E_{\mathrm{f}, \text { geom }}+E_{\mathrm{f} \text {,elec }}=\chi E_{\mathrm{coh}}+\lambda E_{\mathrm{g}}$, where the coefficient $\chi$ is determined by the defect geometries in the spirit of broken-bond models and the coefficient $\lambda$ is related to the electronic properties of defect systems. This linear combination of $E_{\text {coh }}$ and $E_{\mathrm{g}}$ accurately predicts $E_{\mathrm{f}}$ of the point defects not only for Perdew-Burke-Ernzerhof (PBE) [16] and Heyd-Scuseria-Ernzerhof (HSE) [17] family functionals but also for RPA calculations and experiments. Accordingly, $\varepsilon$ can also be understood and predicted by combining $E_{\text {coh }}$ and $E_{\mathrm{g}}$ based on the formulation of $E_{\mathrm{f}}$. We provide evidence that the developed relation holds in a variety of semiconductors, ranging from elemental crystals to compounds, from threedimensional (3D) to two-dimensional (2D) crystals. The two bulk properties used ( $E_{\text {coh }}$ and $\left.E_{\mathrm{g}}\right)$ are easily accessible for a wide range of electronic-structure methods and experiments. Hence, the developed procedure allows us to evaluate the formation energies and charge transition levels of the point defects for RPA and experiments with high accuracy by determining $\chi$ and $\lambda$ from PBE calculations.

\section{METHODS}

All our calculations are performed using the FHI-AIMS code with the "tight" basis set [18]. For each system, we obtain an optimized geometry with PBE and then perform a single-point energy calculation with hybrid functionals [HSE family, PBE0 [19], and PBE0 combined with van der Waals interactions 


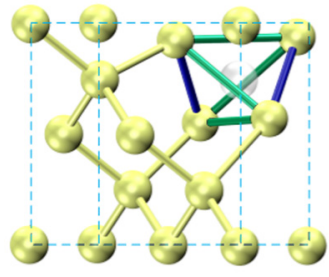

(a)

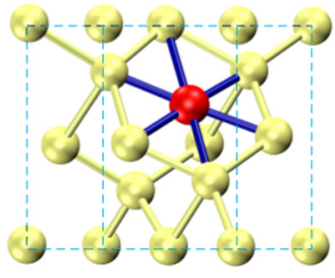

(c)

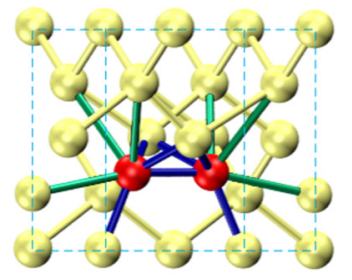

(b)

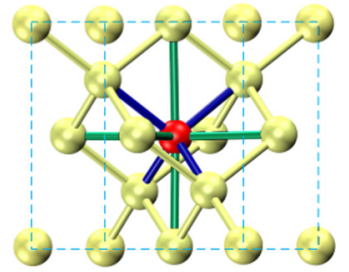

(d)
FIG. 1. Different defect types in a Si supercell, including (a) single vacancy, (b) split $\langle 110\rangle$, (c) hexagonal, and (d) tetrahedral.

(PBE0+vdW) [20-22]]. Here the HSE family consists of HSE, $\mathrm{HSE}_{212}$, and $\mathrm{HSE}_{106}$, the latter two of which are obtained by modulating the mixing fraction of the exact exchange potential $\alpha$ and screening distance $\omega$ of the HSE functional [23]: $\alpha=$ 0.25 and $\omega=0.212 \mathrm{bohr}^{-1}$ for $\mathrm{HSE}_{212} ; \alpha=0.5$ and $\omega=$ $0.106 \mathrm{bohr}^{-1}$ for $\mathrm{HSE}_{106}$. Our extensive tests allow us to adopt a $k$-point grid of $4 \times 4 \times 4$ for a 64 -atom supercell, which converges defect formation energy with an accuracy of $0.01 \mathrm{eV}$.
For all defects, we calculate the formation energy $E_{\mathrm{f}}$ through the equation

$$
E_{\mathrm{f}}=E_{\mathrm{tot}}(\mathrm{D})-E_{\mathrm{tot}}(\mathrm{B})+p_{i} \mu_{i}+q\left(E_{\mathrm{VBM}}+E_{\mathrm{Fermi}}\right),
$$

where $E_{\text {tot }}(\mathrm{D})$ and $E_{\text {tot }}(\mathrm{B})$ are total energies of the defect structure and the pristine bulk (defect free), respectively. $p_{i}$ refers to the number of atoms of type $i$ being removed from or added to the supercell; $p_{i}>0$ means vacancies are created, and $p_{i}<0$ means interstitials are inserted. $\mu_{i}$ is the chemical potential of species $i$ in the reservoir. $q$ denotes the charge state of the defect, $E_{\mathrm{VBM}}$ is the valence-band maximum of the structure with defects, and $E_{\mathrm{Fermi}}$ represents the Fermi level (referenced to $E_{\mathrm{VBM}}$ ). For the formation energy discussed in this work, $E_{\mathrm{Fermi}}$ is set to zero.

\section{RESULTS AND DISCUSSION}

\section{A. Linear combination scheme in elemental semiconductors}

As a demonstration, our focus will be mainly on vacancies and interstitials in Si and Ge, including single-vacancy (SV), double-vacancy (DV), split $\langle 110\rangle(\mathrm{X})$, hexagonal $(\mathrm{H})$, and tetrahedral (T) defects (Fig. 1), given that Si and Ge are among the most studied semiconductors with abundant theoretical and experimental results.

Figure 2 shows the formation energy of vacancies and interstitials in $\mathrm{Si}$ and Ge calculated by $\mathrm{HSE}, \mathrm{HSE}_{212}, \mathrm{HSE}_{106}$, PBE0 [19], and PBE0+vdW [20-22], along with the results of RPA (See Tables S1-S11 in the Supplemental Material [24]); results for both neutral and charged defects are included.
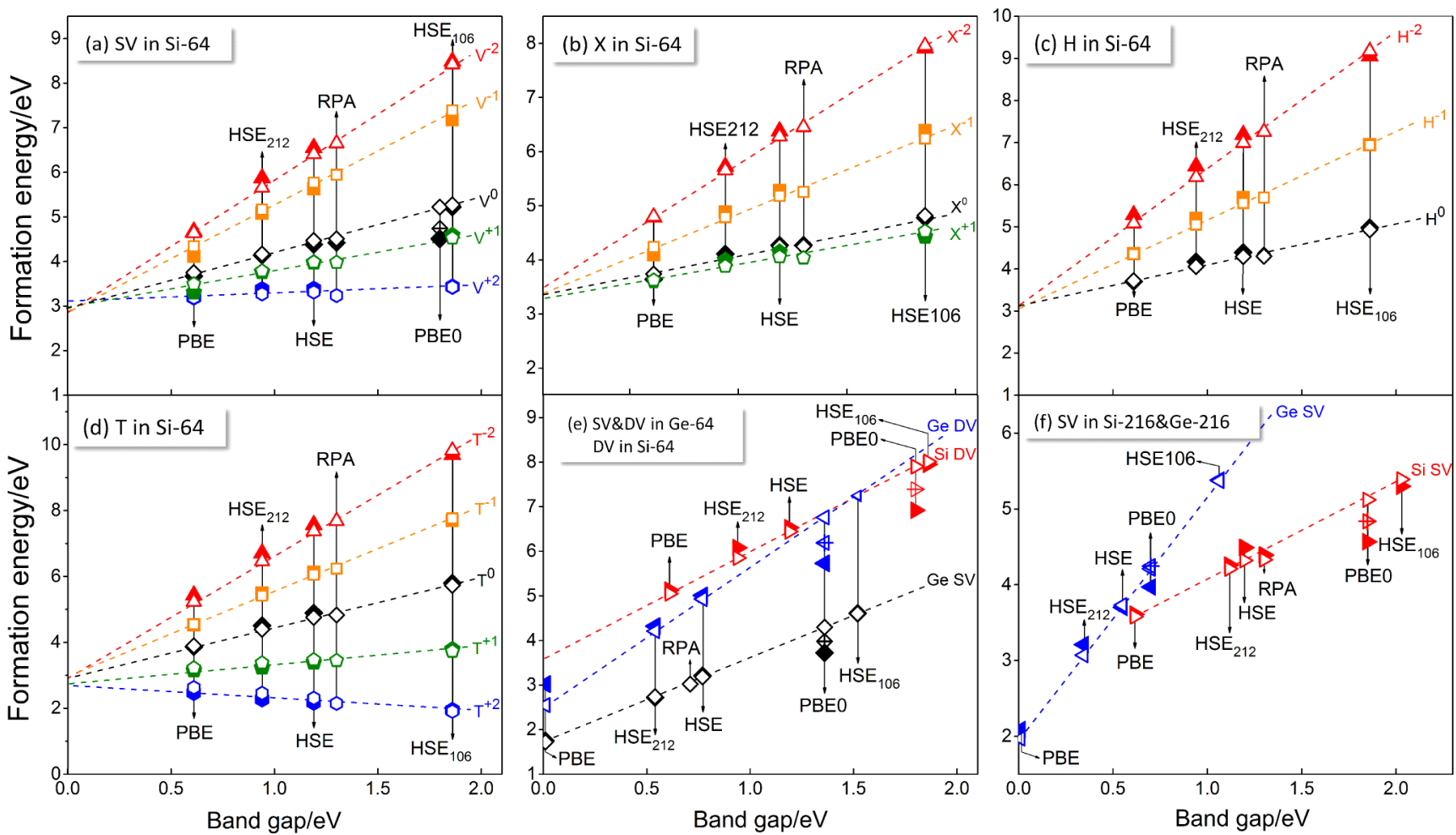

FIG. 2. Formation energy comparison between the calculated values and those predicted by the linear combination of cohesive energy and band gap for Si and Ge. The fully (partially) solid symbols are the calculated results using the five functionals and RPA (PBE0+vdW), while the open symbols are the predicted results. The calculated RPA results and the calculated results for the 216-atom cell are taken from Refs. [14,15], respectively. The dashed lines are based on the values predicted from our linear model. 
Specifically, in a Si 64-atom supercell, the positively charged $\mathrm{X}^{+2}$ and $\mathrm{H}^{+2}$ are relaxed to $\mathrm{T}^{+2}$, whereas the positively charged $\mathrm{H}^{+1}$ is relaxed to $\mathrm{T}^{+1}$. Overall, for each defect, the formation energy increases as follows: $\mathrm{PBE}<\mathrm{HSE}_{212}<$ $\mathrm{HSE}<\mathrm{PBE} 0<\mathrm{HSE}_{106}$ [15], and the formation energy of $\mathrm{PBE}, \mathrm{HSE}, \mathrm{HSE}_{212}$, and $\mathrm{HSE}_{106}$ (excluding PBE0) displays a linear variation with the band gap for each neutral and charged defect in $E_{\mathrm{f}}=\xi+\lambda E_{\mathrm{g}}$. Importantly, the available exact-exchange RPA (EXX-RPA) and range-separated RPA (RS-RPA) results are found to also comply with this linear relationship [14], which motivates us to exploit the underlying physical mechanism behind this remarkably simple behavior.

The band gap and cohesive energy of semiconductors exhibit dramatically different dependence on the mixing fraction of the exact exchange potential $\alpha$ at a fixed value of $\omega$ : the former depends linearly on $\alpha$ [25], whereas the latter is almost independent of $\alpha$. One can tentatively separate the total energy of the bulk into two terms: $E_{\text {tot }}(\alpha)=f_{1}(\alpha)+f_{2}$, with $f_{1}(\alpha)$ determining the band gap and $f_{2}$ determining the cohesive energy. We stress that the total energy does not depend linearly on $\alpha$, which has been confirmed by our results (not shown). The formation energy difference between a certain functional Exchange-Correlation (XC) and PBE at charge state $q$ can be written as $E_{\mathrm{f}}-E_{\mathrm{f}, \mathrm{PBE}}=f_{1}^{\mathrm{d}}(\alpha)-f_{1}(\alpha)-\left(f_{1, \mathrm{PBE}}^{\mathrm{d}}-\right.$ $\left.f_{1, \mathrm{PBE}}\right)+q E_{\mathrm{VBM}}-q E_{\mathrm{VBM}, \mathrm{PBE}}$, while the remaining term, $f_{2}^{\mathrm{d}}-f_{2}-\left(f_{2, \mathrm{PBE}}^{\mathrm{d}}-f_{2, \mathrm{PBE}}\right)$, is approximately equal to zero as $f_{2}$ is independent of $\alpha$. The superscript $\mathrm{d}$ indicates the defect system. For atomically localized defects, semilocal and hybrid schemes generally generate similar wave functions [4] (there are a few exceptions). $f_{1}^{\mathrm{d}}(\alpha)-f_{1}(\alpha)$ can be expressed in a Taylor expansion at $\alpha=0$, whereas VBM scales linearly with $\alpha$ :

$$
\begin{aligned}
E_{\mathrm{f}}-E_{\mathrm{f}, \mathrm{PBE}}= & \left(f_{1, \mathrm{PBE}}^{\mathrm{d}(1)}-f_{1, \mathrm{PBE}}^{(1)}\right) \alpha \\
& +q\left\langle\psi\left|\left(V_{\mathrm{X}, \mathrm{HF}}-V_{\mathrm{X}, \mathrm{PBE}}\right)\right| \psi\right\rangle \alpha,
\end{aligned}
$$

where $V_{\mathrm{X}, \mathrm{HF}}$ and $V_{\mathrm{X}, \mathrm{PBE}}$ are the exact exchange and PBE exchange potential.

Now Eq. (2) can be transformed to a linear combination,

$$
E_{\mathrm{f}}-E_{\mathrm{f}, \mathrm{PBE}}=K_{1} \alpha+K_{2} \alpha,
$$

where coefficients $K_{1}$ and $K_{2}$ equal $f_{1, \mathrm{PBE}}^{\mathrm{d}(1)}-f_{1, \mathrm{PBE}}^{(1)}$ and $q\left\langle\psi\left|\left(V_{\mathrm{X}, \mathrm{HF}}-V_{\mathrm{X}, \mathrm{PBE}}\right)\right| \psi\right\rangle$, respectively. Since the band gap is also proportional to $\alpha$ with $E_{\mathrm{g}}-E_{\mathrm{g}, \mathrm{PBE}}=K_{3} \alpha\left(K_{3}\right.$ is a constant at a fixed value of $\omega$ ) [25], we reach a concise expression for the formation energy:

$$
E_{\mathrm{f}}=\lambda E_{\mathrm{g}}+\xi
$$

where $\lambda=\left(K_{1}+K_{2}\right) / K_{3}$ is dependent on the electron SIE, charge state, and screening distance $\omega . \xi$ is related to cohesive energy because $\xi=f_{2}^{d}-f_{2}$.

Therefore, for functionals like PBE, HSE, and HSE106, each of which has a fixed $\omega$ but different $\alpha$, the formation energies comply with a linear trend. The compliance of HSE212 and EXX-RPA with the linear trend is most likely due to their appropriate compensation between exchange and correlation potentials, while the deviation of PBE0 from the linear trend can be attributed to the full delocalized exchange and the localized correlation in PBE0, which leads to the exchange-correlation functional with incorrect decay

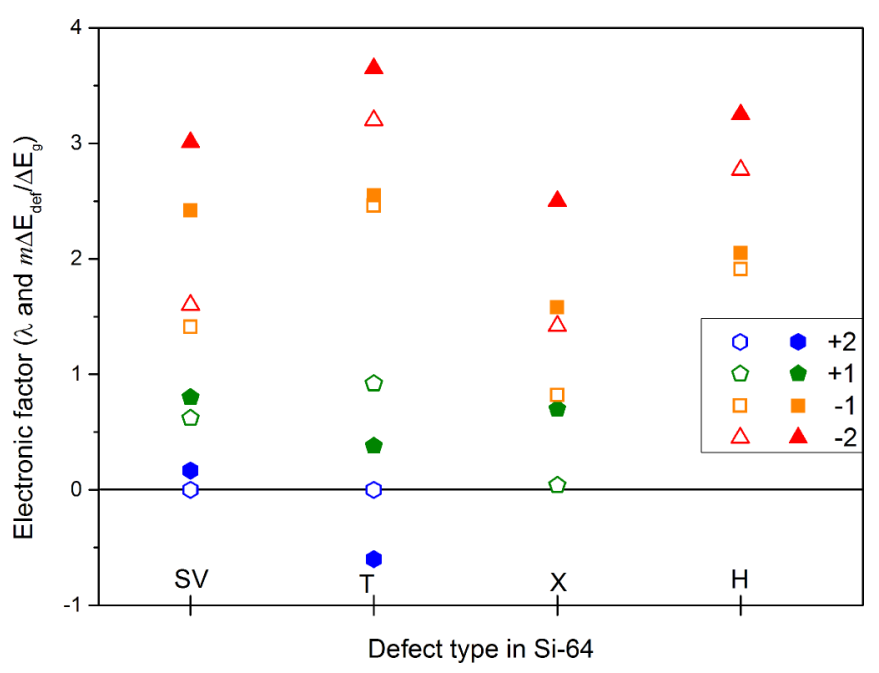

FIG. 3. Comparison between $\lambda$ and $m \Delta E_{\text {def }} / E_{\mathrm{g}}\left(E_{\mathrm{def}}\right.$ is referenced to $E_{\mathrm{VBM}}$ of the defect-free bulk; more details are given in Tables S18 and S19 [24]). The solid symbols are for $\lambda$, while the open symbols are for $m \Delta E_{\mathrm{def}} / E_{\mathrm{g}}$.

in terms of interelectronic distance in semiconductors. The exchange correlation of the EXX-RPA method is combined with exact exchange and the RPA correlation, both of which are delocalized. The semilocal functional PBE is, by construction, based on a local model, having both exchange and correlation functionals localized. Hybrid functionals are constructed by replacing the fraction of PBE localized exchange with delocalized exact exchange. Importantly, only the short-range part of the PBE localized exchange is replaced in range-separated HSE family functionals, while both the short and long ranges of the PBE localized exchange are replaced in PBE0. If part of the long-range correlation, $\mathrm{vdW}$ interactions, is added to PBE0, we obtain the formation energy of PBE0+vdW, which is in better agreement with the values predicted by the fitted relation of $E_{\mathrm{f}}=\xi+\lambda E_{\mathrm{g}}$ for SVs and DVs of Si and $\mathrm{Ge}$ (Fig. 2). These results confirm that the comparably localized nature of the exchange-correlation functional in PBE, HSE family functionals, and EXX-RPA is essential to their identical coefficient $\lambda$.

Importantly, our proposed relationship between formation energy and band gap is significantly different from the scheme based on the linear dependence of the occupied defect level with different level methods. By adding the shift term $m \Delta E_{\text {def }}$ ( $m$ is the occupation number, and $\Delta E_{\mathrm{def}}$ is the shift of the defect level) to the formation energy of the semilocal functional results, one could approximate the formation energy of hybrid functionals for some neutral defects [26]. However, this scheme does not produce satisfying results for most defect cases. Whether $E_{\text {def }}$ is referenced to $E_{\mathrm{VBM}}$ of the defect-free bulk or the structure with defects, the slopes obtained with the shift defect-level scheme $\left(m \Delta E_{\text {def }} / \Delta E_{\mathrm{g}}\right)$ clearly contradict our results for $\lambda$. For the charged vacancies and interstitials, the deviation is up to $600 \%$ (Fig. 3 and Tables S18 and S19 in the Supplemental Material [24]). For the example of $V^{+2}$ and $T^{+2}(\lambda=0.167$ and -0.6$)$, the shifting scheme cannot capture the variation of $E_{\mathrm{f}}$ at all because the occupation number of the defect level is zero for both defects (leading to $m \Delta E_{\mathrm{def}} / \Delta E_{\mathrm{g}}=0$ ). 
We now turn to analyzing the physical meaning of the intercept $\xi$ in the fitted relation of $E_{\mathrm{f}}=\xi+\lambda E_{\mathrm{g}}$ by taking point defects in $\mathrm{Si}$ as the example elemental semiconductor. The creation of SVs and interstitials in Si is accompanied by breaking and forming $\mathrm{Si}-\mathrm{Si}$ bonds, which is naturally related to broken-bond models. It has been established in broken-bond models that the energy cost for breaking a bond is proportional to $E_{\mathrm{coh}} / Z_{\mathrm{B}}$ for strong covalent bonds but $\left(\sqrt{Z_{\mathrm{B}}}-\sqrt{Z_{\mathrm{B}}-1}\right) E_{\mathrm{coh}} / \sqrt{Z_{\mathrm{B}}}$ for relatively weak bonds [27] ( $E_{\mathrm{coh}}$ is defined as a positive value for convenience), where $Z_{\mathrm{B}}$ is the coordination number of the bulk. The creation of SVs breaks strong covalent Si-Si bonds; in stark contrast, the creation of interstitials forms extraweak $(\mathrm{Si}-\mathrm{Si})_{I}$ bonds since each bulk $\mathrm{Si}$ atom resembles the electron cloud of a noble gas with $Z_{\mathrm{B}}=4$. We can thus evaluate the geometric energy cost for generating vacancies and interstitials, which is proportional to $E_{\text {coh }} / 4$ and $(\sqrt{5}-2) E_{\text {coh }}^{\prime} / \sqrt{5}=(\sqrt{5}-2) E_{\mathrm{coh}} / 2$, respectively, where $E_{\text {coh }}^{\prime}$ is the cohesive energy of a $\mathrm{Si}$ atom (around the interstitial atom) with $Z_{\mathrm{B}}^{\prime}=5$. For simplification, we define $E_{\text {coh }}$ of an interstitial to be $\left(Z_{\mathrm{B}}^{\prime} E_{\text {bond }}^{\prime}\right) / 2$, with the coordination number $Z_{\mathrm{B}}^{\prime}$ of the defect center atom and the bond energy of the interstitial $E_{\text {bond }}^{\prime}$. It is known that broken-bond models do not correctly capture the effect of relaxation owing to the lack of exact geometric input (e.g., rebinding of dangling atoms and changing of bond length). This can be readily remedied by scaling the bond length of the newly formed $\mathrm{Si}-\mathrm{Si}$ bonds $L_{i}$ with respect to the bulk Si-Si bonds $L$. The geometric energy $E_{\mathrm{f} \text {,geom }}$ cost to create SVs and interstitials is defined as follows:

$$
\begin{aligned}
& E_{\mathrm{f}, \text { geom }}(V)=\left(1-\sum_{i} n_{i} \kappa_{i} / 4\right) E_{\mathrm{coh}}, \\
& E_{\mathrm{f}, \text { geom }}(I)=\sum_{i} n_{i} \kappa_{i}(\sqrt{5} / 2-1) E_{\mathrm{coh}},
\end{aligned}
$$

where $n_{i}$ is the number of newly formed bonds with the bond length $L_{i}$ as the defects are created ( $i$ denotes the order of the neighbor shell around the defect site) and $\kappa_{i}$ is the bond strength ratio between the newly formed bonds and the bulk bonds. The ratio $\kappa_{i}$ is a function of bond length, and we assume it to be determined by the inversely proportional relationship $[28,29]$ or by the Lennard-Jones potential. The former is defined as $\kappa_{i}=L_{i} / L$; our results indicate it is particularly suitable for vacancies in $\mathrm{Si}, \mathrm{InP}$, and phosphorenes in which the dangling atoms form relatively strong bonds, e.g., through Jahn-Teller (JT) distortion. Conversely, the Lennard-Jones potential, which is widely used to describe the attractive vdW potential, is sufficiently appropriate for the relatively weak bonds such as interstitials in $\mathrm{Si}$ and the other considered vacancies, with $\kappa_{i}=2\left(L / L_{i}\right)^{6}-\left(L / L_{i}\right)^{12}$. Notably, the interaction between the $\mathrm{T}$ defect atom and its second-nearest neighbors is half of that between $\mathrm{T}$ and its nearest neighbors [30].

We compute $E_{\mathrm{f}, \text { geom }}$ for SVs, DVs, and interstitials of $\mathrm{Si}$ and Ge with 64-atom supercells, summarizing the results in Table I, Fig. 2, and Tables S1-S11 [24]. Given that the cohesive energy deviates less than $0.05 \mathrm{eV}$ from PBE to the HSE family of functionals, $E_{\mathrm{f} \text {,geom values from different functionals }}$ are almost identical for the same defect, which remarkably
TABLE I. Electronic factor $\lambda$ and geometric factors $n_{i} / L_{i}$ and $\chi$ for calculating $E_{\mathrm{f}}$ and the geometric contribution $E_{\mathrm{f}, \text { geom }}$ of $\mathrm{SVs}$, DVs, and interstitials of Si with a 64-atom supercell $\left(L_{\mathrm{Si} \text {,bulk }}=2.37 \AA\right.$ ). The fitting geometric contribution $\xi$ is shown for comparison, while the predicted values based on EXX-RPA (P-EXX-RPA), RS-RPA (P-RS-RPA), and experiment (P-Exp) computed or measured $E_{\text {coh }}$ and $E_{\mathrm{g}}$ are compared to the direct calculations or measurements, respectively.

\begin{tabular}{lccccc}
\hline \hline & $\mathrm{SV}$ & $\mathrm{X}$ & $\mathrm{H}$ & $\mathrm{T}$ & $\mathrm{DV}$ \\
\hline$\lambda$ & 1.20 & 0.85 & 0.95 & 1.45 & 2.3 \\
$n_{1} / L_{1}$ & $2 / 3.61$ & $3 / 2.49$ & $6 / 2.42$ & $4 / 2.48$ & $6 / 3.37$ \\
$n_{2} / L_{2}$ & & $6 / 2.86$ & & $6 / 2.77$ & \\
$\chi$ & 0.672 & 0.715 & 0.699 & 0.669 & 0.805 \\
$E_{\mathrm{f}, \text { geom }}$ & 3.02 & 3.21 & 3.14 & 3.00 & 3.61 \\
$\xi$ & 3.00 & 3.20 & 3.17 & 3.03 & 3.88 \\
P-EXX-RPA & 4.51 & 4.24 & 4.30 & 4.82 & 6.59 \\
EXX-RPA [14] & 4.42 & 4.27 & 4.37 & 4.84 & \\
P-RS-RPA & 4.26 & 4.42 & 4.48 & 4.99 & 6.79 \\
RS-RPA [13] & 4.24 & 4.49 & 4.74 & & \\
P-Exp & 4.09 & 4.30 & 4.34 & 4.79 & 6.47 \\
Expt. [31-35] & $4.0,3.6 \pm 0.2$ & & $4.2-4.7$ & & \\
\hline \hline
\end{tabular}

resembles the intercept $\xi$ of $E_{\mathrm{f}}=\xi+\lambda E_{g}$, with the largest deviations of $0.03 \mathrm{eV}$ for neutral SVs and interstitials $(<1 \%)$ and $0.27 \mathrm{eV}$ for DVs $(<7 \%)$. This consistency also holds for charged SVs and interstitials in Si (Tables S7-S11 [24]) and neutral SVs and interstitials in Si and Ge with 216atom supercells (calculated using the VASP code in Fig. 2) $[15,36]$. Therefore, the intercept $\xi$ is essentially the geometric contribution of the defect formation energy: $\xi=E_{\mathrm{f}, \mathrm{geom}}=$ $\chi E_{\text {coh }}$ (the intercept of the dashed lines in Fig. 2).

By now we have shown the procedure for computing $\chi$ and $E_{\text {geom }}$. The electronic contribution $\lambda E_{g}$ can then be obtained by subtracting $E_{\text {geom }}$ from $E_{\mathrm{f}}$. Therefore, one PBE calculation is able to generate information for both $\chi$ and $\lambda$. We compare the $\lambda$ obtained with PBE to that obtained with linear fitting for neutral vacancy, $\mathrm{X}, \mathrm{H}$, and $\mathrm{T}$ defects of $\mathrm{Si}$, as well as the O SV in $\mathrm{ZnO}$. The difference between them is typically within $6 \%$ (see Table II), indicating the efficiency and reliability of the scheme. The same applies to the charged defects. We find that $\lambda$ is dependent on the charge states but is independent of the position of $E_{\text {Fermi }}$ for a defect with a particular charge state $q$. The identified $\lambda$ would always work at any Fermi level since

TABLE II. Comparison between $\lambda$ obtained with a single PBE calculation (denoted as $\lambda_{1}$ ) and linear fitting to different functionals (denoted as $\lambda_{2}$ ). Here Si-64 and Si-216 indicate Si with 64- and 216-atom supercells.

\begin{tabular}{lcc}
\hline \hline & $\lambda_{1}$ & $\lambda_{2}$ \\
\hline SV in Si-64 & 1.07 & 1.20 \\
X in Si-64 & 0.70 & 0.85 \\
H in Si-64 & 0.92 & 0.95 \\
T in Si-64 & 1.42 & 1.45 \\
DV in Si-64 & 2.48 & 2.35 \\
SV in Si-216 & 1.30 & 1.26 \\
O SV in ZnO (O rich) & 0.38 & 0.40 \\
\hline \hline
\end{tabular}


the change in Fermi level $\Delta E_{\mathrm{Fermi}}$ just adds a value $q \Delta E_{\mathrm{Fermi}}$ to the formation energy of each functional, which does not change $\lambda$.

We then apply the expression of $E_{\mathrm{f}}=\chi E_{\mathrm{coh}}+\lambda E_{\mathrm{g}}$ with the EXX-RPA $E_{\text {coh }}$ and $E_{\mathrm{g}}$ to $\mathrm{Si}$; we predict the formation energies of SVs and interstitials for a $\mathrm{Si}$ 64-atom supercell (Fig. 2 and Table I), which are in excellent agreement with the explicitly calculated EXX-RPA results of Kresse et al. [14], with a deviation $<3 \%(0.09 \mathrm{eV}$ for $\mathrm{SVs}$ and $<0.07 \mathrm{eV}$ for interstitials). This conclusion also holds for $\mathrm{SVs}$ in a $\mathrm{Si}$ 216-atom supercell [14,15] [Fig. 2(f)]. Therefore, our scheme allows an accurate determination of defect formation energies for EXX-RPA based on PBE calculations. This identified relation between $E_{\mathrm{f}}$ and the bulk properties of $E_{\mathrm{coh}} / E_{\mathrm{g}}$ can also be used to predict results based on experimental measurements, yielding defect formation energies that are consistent with experimental values, in particular for point defects in $\mathrm{Si}$ (Table I).

It is noteworthy that an accurate prediction of $E_{\mathrm{f}}$ relies on the correct description of geometric structure; therefore, calculation models and methods will affect predictions. As shown in Figs. 2(e) and 2(f), the predicted $E_{\mathrm{f}}$ for $\mathrm{SV}$ in a Ge 64-atom supercell has a smaller intercept than that in a Ge 216-atom supercell. Due to the finite-size effect, the former has shorter and stronger newly formed bonds; thus, the geometric factor $\chi$ and geometric contribution are smaller (while the electron SIE and/or screening distance $\omega$ differences between the different supercells also lead to a smaller electronic factor $\lambda$ and electronic contribution in a $\mathrm{Ge} 64$-atom supercell). In the case of RS-RPA results based on local-density approximation (LDA) JT geometries, the LDA JT geometries generate a smaller coefficient $\chi$ relative to our PBE geometries ( 0.583 vs 0.672 in a 64-atom supercell with $n_{1} / L_{1}=2 / 2.82$ ), yielding the RS-RPA formation energy with $E_{\mathrm{f}}=\chi E_{\text {coh }}+\lambda E_{\mathrm{g}}$, in good agreement with the direct RS-RPA calculations (4.26 vs $4.24 \mathrm{eV}$ ) [13]. It is known that PBE fails to describe the JT distortion of a SV in Si with a 64-atom supercell [13,22], predicting the undistorted SV to be more stable by $0.04 \mathrm{eV}$. Nevertheless, this failure can be corrected by applying our scheme using the experimental $E_{\text {coh }}$ and $E_{\mathrm{g}}$ : the predicted $E_{\mathrm{f}}$ is $4.51 \mathrm{eV}$ for the undistorted SV $(\chi=0.672)$ and $4.09 \mathrm{eV}$ for the JT distorted SV $(\chi=0.583)$, consistent with the RPA results [13].

\section{B. Generalization of the linear combination scheme in a 2D crystal and binary semiconductor}

We also generalize our conclusions by correlating the linear combination of $E_{\text {coh }}$ and $E_{\mathrm{g}}$ to the formation energy of SVs in phosphorenes, $\mathrm{ZnO}, \mathrm{GaAs}$, and InP. Phosphorenes, which have four phases, $\alpha, \beta, \gamma$, and $\delta$, with distinct band gaps, are representative of $2 \mathrm{D}$ materials [37]. For a compound semiconductor, the formation energy of a point defect is no longer a fixed value but varies with the reference one chooses for the chemical potential $\mu$ of the defect atom (Fig. 4). Take the Ga vacancy in GaAs as an example $\left(V_{\mathrm{Ga}}\right)$; the difference between the formation energies of the two limiting conditions (Ga rich and As rich) is as follows:

$$
E_{\mathrm{f}}^{\text {Garich }}-E_{\mathrm{f}}^{\text {Asrich }}=\mu_{\mathrm{Ga}}^{\text {Garich }}-\mu_{\mathrm{As}}^{\text {Asrich }}
$$

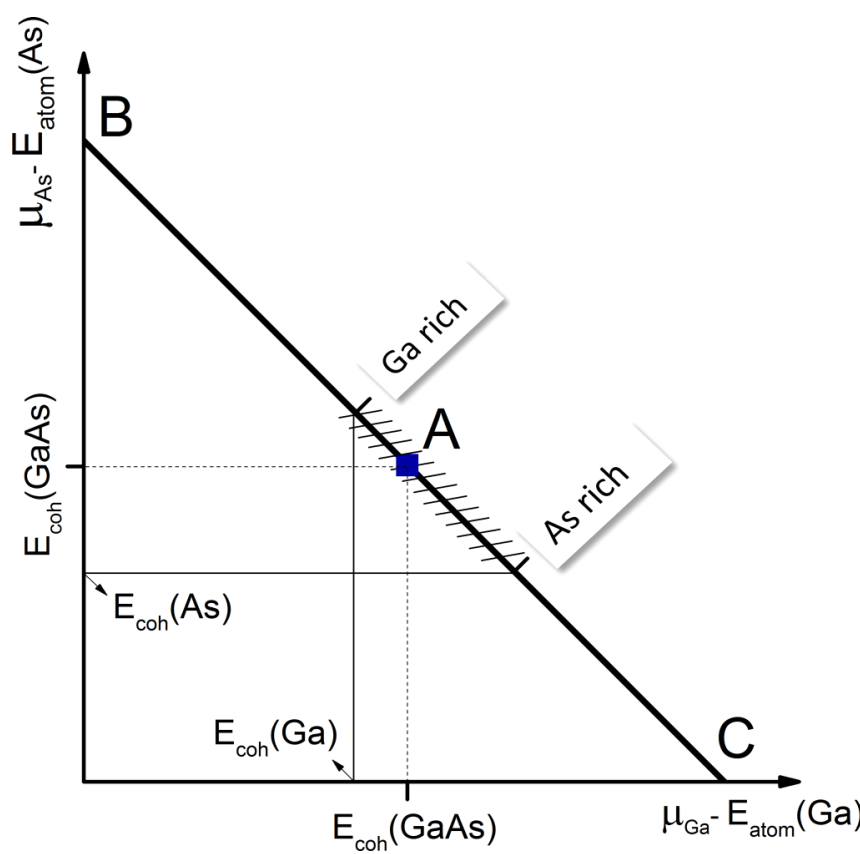

FIG. 4. Thermodynamic stable range for equilibrium growth of $\mathrm{GaAs}$ (hatched part along line $\mathrm{BC}$ ); the relative chemical potentials of $\mathrm{Ga}$ and $\mathrm{As}$ atoms are equal at point $\mathrm{A}$. The coordination is ( $\left.E_{\mathrm{coh}}(\mathrm{GaAs}), \mathrm{E}_{\mathrm{coh}}(\mathrm{GaAs})\right)$ for point $\mathrm{A},\left(0,2 E_{\mathrm{coh}}(\mathrm{GaAs})\right)$ for point $\mathrm{B}$, and $\left(2 E_{\mathrm{coh}}(\mathrm{GaAs}), 0\right)$ for point $\mathrm{C}$.

because $\quad \mu_{\mathrm{Ga}}^{\mathrm{Garich}}=E_{\mathrm{atom}}(\mathrm{Ga})-E_{\mathrm{coh}}(\mathrm{Ga}) \quad$ and $\quad \mu_{\mathrm{Ga}}^{\text {Asrich }}=$ $\mu_{\mathrm{GaAs}}-\mu_{\mathrm{As}}^{\mathrm{Asrich}}=E_{\mathrm{atom}}(\mathrm{Ga})+E_{\mathrm{coh}}(\mathrm{As})-2 E_{\mathrm{coh}}(\mathrm{GaAs})$, where $E_{\text {atom }}$ represents the energy of an isolated atom. Substituting these equations into Eq. (7), we obtain

$$
E_{\mathrm{f}}^{\text {Garich }}-E_{\mathrm{f}}^{\text {Asrich }}=2 E_{\mathrm{coh}}(\mathrm{GaAs})-E_{\mathrm{coh}}(\mathrm{As})-E_{\mathrm{coh}}(\mathrm{Ga}) \text {. }
$$

As cohesive energies are $\alpha$ insensitive, the difference between $E_{\mathrm{f}}^{\text {Garich }}$ and $E_{\mathrm{f}}^{\text {Asrich }}$ does not vary with $E_{\mathrm{g}}$; therefore, we ascribe this term to the $E_{\text {geom }}$ part:

$$
E_{\text {geom }}^{\text {Garich }}\left(V_{\mathrm{Ga}}\right)-E_{\text {geom }}^{\text {Asrich }}\left(V_{\mathrm{Ga}}\right)=\mu_{\mathrm{Ga}}^{\text {Garich }}-\mu_{\mathrm{Ga}}^{\text {Asrich }} .
$$

To draw an analogy to the elemental semiconductor, we transform Eq. (9) into

$$
E_{\text {geom }}^{\mathrm{Garich}}\left(V_{\mathrm{Ga}}\right)-E_{\text {geom }}^{\prime}\left(V_{\mathrm{Ga}}\right)=\mu_{\mathrm{Ga}}^{\mathrm{Garich}}-\mu_{\mathrm{Ga}}^{\prime} .
$$

Here $E_{\text {geom }}^{\prime}\left(V_{\mathrm{Ga}}\right)$ is the geometric part of $E_{\mathrm{f}}$ for the GaAs compound under average conditions, where the $\mathrm{Ga}$ atom and the As atom have the same energy gain when forming the solid:

$$
\mu_{\mathrm{Ga}}^{\prime}=E_{\text {atom }}(\mathrm{Ga})-E_{\mathrm{coh}}(\mathrm{GaAs})
$$

and

$$
\mu_{\text {As }}^{\prime}=E_{\text {atom }}(\mathrm{As})-E_{\text {coh }}(\mathrm{GaAs}) .
$$

Then the difference in geometric contributions between the Ga-rich condition and the average condition is

$$
E_{\text {geom }}^{\text {Garich }}\left(V_{\mathrm{Ga}}\right)-E_{\text {geom }}^{\prime}\left(V_{\mathrm{Ga}}\right)=E_{\mathrm{coh}}(\mathrm{GaAs})-E_{\mathrm{coh}}(\mathrm{Ga}) \text {. }
$$

This average condition can be achieved by tuning the growth environment. As shown in Fig. 4, at point A, Ga and As atoms have the same relative chemical potential equaling 

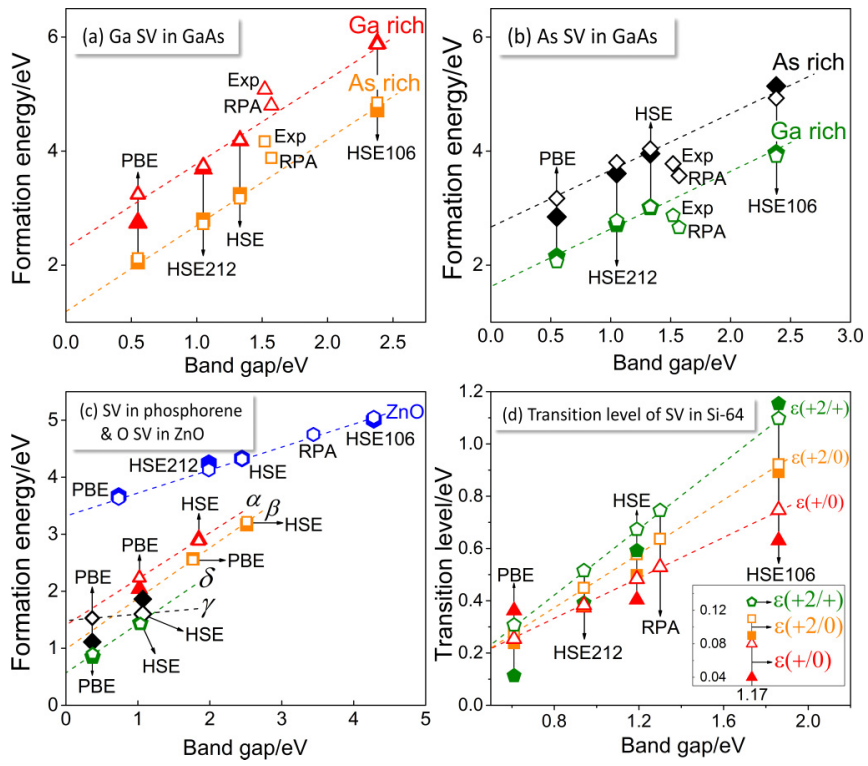

FIG. 5. Formation energy and charge transition level comparison between the calculated values and those predicted by the linear combination of cohesive energy and band gap for phosphorenes, $\mathrm{ZnO}$, $\mathrm{GaAs}, \mathrm{InP}$, and Si. The inset in (d) shows the results of Si extrapolated to infinite size. The solid symbols are the results calculated by the four functionals, while the open symbols are the predicted results. The calculated results for $\mathrm{ZnO}$ are taken from Ref. [15]. The dashed lines are based on the predicted value.

to $E_{\text {coh }}(\mathrm{GaAs})$. At this point, we treat the compound as an elemental crystal by using the same method to calculate geometric contributions in $\mathrm{Si}$ and $\mathrm{Ge}$ :

$$
E_{\text {geom }}^{\prime}\left(V_{\mathrm{Ga}}\right)=\left(1-\sum_{i} n_{i} \kappa_{i} / 4\right) E_{\mathrm{coh}}(\mathrm{GaAs}) .
$$

Consequently, the geometric contribution of the Ga-rich type is

$$
\begin{aligned}
E_{\mathrm{geom}}^{\mathrm{Garich}}\left(V_{\mathrm{Ga}}\right)= & \left(1-\sum_{i} n_{i} \kappa_{i} / 4\right) E_{\mathrm{coh}}(\mathrm{GaAs}) \\
& +E_{\mathrm{coh}}(\mathrm{GaAs})-E_{\mathrm{coh}}(\mathrm{Ga}) .
\end{aligned}
$$

Similarly, the geometric contribution of the As-rich type is

$$
\begin{aligned}
E_{\mathrm{geom}}^{\text {Asrich }}\left(V_{\mathrm{Ga}}\right)= & \left(1-\sum_{i} n_{i} \kappa_{i} / 4\right) E_{\mathrm{coh}}(\mathrm{GaAs}) \\
& +E_{\mathrm{coh}}(\mathrm{As})-E_{\mathrm{coh}}(\mathrm{GaAs}) .
\end{aligned}
$$

It is noteworthy that the condition of point $\mathrm{A}$ is not always favorable for the growth of the compound, which may be outside the thermodynamic stable range (line BC in Fig. 4) in some cases. In fact, it is treated as an ideal model connecting the elemental crystal and compound.

In all considered cases, the formation energies predicted by the linear combination of $E_{\mathrm{coh}}$ and $E_{\mathrm{g}}$ provide an excellent fit for the ones calculated with PBE and HSE family functionals [15] (see Fig. 5 and Tables S14-S17 [24]). Thus, the relation between cohesive energy/band gap and formation energy is a rather general phenomenon in semiconductors, including
3D elemental crystals, 3D compounds, and 2D crystals. Based on this correlation, we also predict the formation energy of SVs for phosphorenes, $\mathrm{ZnO}, \mathrm{GaAs}$, and InP with the available RPA and experimental $E_{\text {coh }} / E_{\mathrm{g}}$ [38-42]. The predicted $E_{\mathrm{f}}$ of a Ga SV of GaAs in the As-rich condition is $4.17 \mathrm{eV}$, in good agreement with experimentally measured $E_{\mathrm{f}}, 4.0 \pm 0.5 \mathrm{eV}[43]$.

\section{Linear combination scheme for defect transition levels}

Finally, we attempt to connect the defect charge transition level $\varepsilon\left(q / q^{\prime}\right)$ (defined with respect to the VBM) to the bulk properties of the host, obtaining the relation $\varepsilon\left(q / q^{\prime}\right)=\left[\left(\lambda^{\prime}-\lambda\right) E_{\mathrm{g}}-\left(\chi^{\prime}-\chi\right) E_{\mathrm{coh}}\right] /\left(q-q^{\prime}\right) . \quad$ Namely, the defect charge transition level also consists of geometric and electronic contributions. Overall, the predictions of $\varepsilon\left(q / q^{\prime}\right)$ with a combination of $E_{\mathrm{coh}}$ and $E_{\mathrm{g}}$ are in reasonable agreement with the directly calculated values for SVs and interstitials, with the deviation mostly around $10 \%$ in a Si 64-atom supercell [Fig. 5(d) and Tables S12 and S13 [24]]. Notably, the predictions by our scheme recover the "negative- $U$ " effect of SVs (that the stable charge state of a $\mathrm{SV}$ changes from $\mathrm{V}^{+2}$ to $\mathrm{V}^{0}$ directly, where $\mathrm{V}$ indicates a vacancy) in a $\mathrm{Si}$ 64-atom supercell for all functionals used, which cannot be properly described by the direct PBE calculations in such a small supercell. The geometric contribution to $\varepsilon\left(q / q^{\prime}\right)$ is up to $0.15 \mathrm{eV}$ for the interstitials and SVs of Si due to the change in defect geometry induced by increasing electronic charge. Since finite-size effects can significantly affect charged defect calculations, we calculated SVs of Si in a 216-atom supercell and extrapolated the results to infinite size [inset of Fig. 5(d)], predicting experimental charge transition levels of $\varepsilon(+2 /+)=0.13, \varepsilon(+2 / 0)=0.11$, and $\varepsilon(+/ 0)=0.08 \mathrm{eV}$, which are in agreement with the well-established experimental results of $\varepsilon(+2 /+)=$ 0.13, $\varepsilon(+2 / 0)=0.09, \quad$ and $\quad \varepsilon(+/ 0)=0.03-0.05 \quad \mathrm{eV}$ [44]. Our predicted RPA results for $\varepsilon\left(q / q^{\prime}\right)$ (Tables $\mathrm{S} 12$ and S13 [24]) also provide useful references for further RPA studies on defects.

\section{CONCLUSIONS}

In summary, we proposed a model for the defect formation energy and transition energy levels of point defects in semiconductors. Defect formation energies and charge transition levels were found to depend on the cohesive energy and band gap of host materials with the relations $E_{\mathrm{f}}=\chi E_{\mathrm{coh}}+\lambda E_{\mathrm{g}}$ and $\varepsilon\left(q / q^{\prime}\right)=\left[\left(\lambda^{\prime}-\lambda\right) E_{\mathrm{g}}-\left(\chi^{\prime}-\chi\right) E_{\mathrm{coh}}\right] /\left(q-q^{\prime}\right)$, where $\chi / \chi^{\prime}$ and $\lambda / \lambda^{\prime}$ are accessible by the geometric and electronic factors of defects, respectively. We found that these correlations are applicable to a variety of semiconductors ranging from elemental crystals to compounds, from 3D to $2 \mathrm{D}$ crystals, remarkably enabling the determination of defect formation energies and charge transition levels for RPA and experiments based on (semi)local DFT calculations. Our results demonstrate that the screened hybrid HSE functional outperforms (semi)local and full hybrid functionals in calculating formation energies and charge transition levels of point defects, supporting the practice of matching experimental band gap values by optimizing HSE (but not PBE0) parameters. 


\section{ACKNOWLEDGMENTS}

We gratefully acknowledge support from the Thousand Young Talents Plan, the National Natural Science Foundation of China (No. 21673095, 51631004), and the computing resources of High Performance Computing Center of Jilin University, National Supercomputing Center in Jinan, and in Tianjin China.
[1] C. Freysoldt, B. Grabowski, T. Hickel, J. Neugebauer, G. Kresse, A. Janotti, and C. G. Van de Walle, Rev. Mod. Phys. 86, 253 (2014).

[2] S. Lany and A. Zunger, Phys. Rev. B 78, 235104 (2008).

[3] P. Deák, A. Gali, B. Aradi, and T. Frauenheim, Phys. Status Solidi B 248, 790 (2011).

[4] A. Alkauskas, P. Broqvist, and A. Pasquarello, Phys. Rev. Lett. 101, 046405 (2008)

[5] A. Janotti, J. B. Varley, P. Rinke, N. Umezawa, G. Kresse, and C. G. Van de Walle, Phys. Rev. B 81, 085212 (2010).

[6] H.-P. Komsa, P. Broqvist, and A. Pasquarello, Phys. Rev. B 81, 205118 (2010).

[7] A. Alkauskas, P. Broqvist, and A. Pasquarello, Phys. Status Solidi B 248, 775 (2011).

[8] P. Rinke, A. Janotti, M. Scheffler, and C. G. Van de Walle, Phys. Rev. Lett. 102, 026402 (2009).

[9] A. E. Mattsson, R. R. Wixom, and R. Armiento, Phys. Rev. B 77, 155211 (2008).

[10] W.-K. Leung, R. J. Needs, G. Rajagopal, S. Itoh, and S. Ihara, Phys. Rev. Lett. 83, 2351 (1999).

[11] E. R. Batista, J. Heyd, R. G. Hennig, B. P. Uberuaga, R. L. Martin, G. E. Scuseria, C. J. Umrigar, and J. W. Wilkins, Phys. Rev. B 74, 121102 (2006).

[12] W. D. Parker, J. W. Wilkins, and R. G. Hennig, Phys. Status Solidi B 248, 267 (2011).

[13] F. Bruneval, Phys. Rev. Lett. 108, 256403 (2012).

[14] M. Kaltak, J. Klimeš, and G. Kresse, Phys. Rev. B 90, 054115 (2014).

[15] R. Ramprasad, H. Zhu, P. Rinke, and M. Scheffler, Phys. Rev. Lett. 108, 066404 (2012).

[16] J. P. Perdew, K. Burke, and M. Ernzerhof, Phys. Rev. Lett. 77, 3865 (1996).

[17] J. Heyd, G. E. Scuseria, and M. Ernzerhof, J. Chem. Phys. 124, 219906 (2006).

[18] V. Blum, R. Gehrke, F. Hanke, P. Havu, V. Havu, X. Ren, K. Reuter, and M. Scheffler, Comput. Phys. Commun. 180, 2175 (2009).

[19] J. P. Perdew, M. Ernzerhof, and K. Burke, J. Chem. Phys. 105, 9982 (1996)

[20] G.-X. Zhang, A. Tkatchenko, J. Paier, H. Appel, and M. Scheffler, Phys. Rev. Lett. 107, 245501 (2011).

[21] A. Tkatchenko, R. A. DiStasio, Jr., R. Car, and M. Scheffler, Phys. Rev. Lett. 108, 236402 (2012).
[22] W. Gao and A. Tkatchenko, Phys. Rev. Lett. 111, 045501 (2013).

[23] A. V. Krukau, O. A. Vydrov, A. F. Izmaylov, and G. E. Scuseria, J. Chem. Phys. 125, 224106 (2006).

[24] See Supplemental Material at http://link.aps.org/supplemental/ 10.1103/PhysRevB.96.245203 for detailed data for formation energy and full calculation procedures.

[25] F. Viñes, R. Lamiel-García, K. C. Ko, J. Y. Lee, and F. Illas, J. Comput. Chem. 38, 781 (2017).

[26] R. Mishra, O. D. Restrepo, A. Kumar, and W. Windl, J. Mater Sci. 47, 7482 (2012).

[27] M. Methfessel, D. Hennig, and M. Scheffler, Phys. Rev. B 46, 4816 (1992).

[28] A. Aresti, L. Garbato, and A. Rucci, J. Phys. Chem. Solids 45, 361 (1984).

[29] H. Schlosser, Phys. Status Solidi B 179, K1 (1993).

[30] Q. Jiang, H. M. Lu, and M. Zhao, J. Phys. Condens. Matter 16, 521 (2004).

[31] N. Fukata, A. Kasuya, and M. Suezawa, Physica B (Amsterdam, Neth.) 308, 1125 (2001).

[32] S. Dannefaer, P. Mascher, and D. Kerr, Phys. Rev. Lett. 56, 2195 (1986).

[33] H. Bracht, N. A. Stolwijk, and H. Mehrer, Phys. Rev. B 52, 16542 (1995).

[34] H. Bracht, E. E. Haller, and R. Clark-Phelps, Phys. Rev. Lett. 81, 393 (1998).

[35] A. Ural, P. B. Griffin, and J. D. Plummer, Phys. Rev. Lett. 83, 3454 (1999).

[36] G. Kresse and J. Furthmüller, Phys. Rev. B 54, 11169 (1996).

[37] J. Guan, Z. Zhu, and D. Tománek, Phys. Rev. Lett. 113, 046804 (2014).

[38] J. Harl, L. Schimka, and G. Kresse, Phys. Rev. B 81, 115126 (2010).

[39] J. Klimeš and G. Kresse, J. Chem. Phys. 140, 054516 (2014).

[40] H. Liu, A. T. Neal, Z. Zhu, Z. Luo, X. F. Xu, D. Tománek, and P. D. Ye, ACS Nano 8, 4033 (2014).

[41] C. Kittel, Introduction to Solid State Physics, 8th ed. (Wiley, Hoboken, NJ, 2004).

[42] J. Uddin and G. E. Scuseria, Phys. Rev. B 74, 245115 (2006).

[43] J.-L. Rouviere, Y. Kim, J. Cunningham, J. A. Rentschler, A. Bourret, and A. Ourmazd, Phys. Rev. Lett. 68, 2798 (1992).

[44] G. D. Watkins, Deep Centres in Semiconductors (Gordon and Breach, Yverdon, Switzerland, 1986), p. 147. 